\title{
Cross-correlation of X-rays for 4U 1608-52
}

\author{
Y. J. Lei, H. T. Zhang, A. L. Luo and Y. H. Zhao \\ National Astronomical Observatories, Chinese Academy of Sciences, Beijing, China \\ email: leiyj cwmy@163.com
}

\begin{abstract}
We study the cross-correlation between X-rays of different energies for the atolltype source $4 \mathrm{U}$ 1608-52 with RXTE, and find the cross-correlation evolutes along the different branches. The anti-correlation is reported from the Galactic black hole candidates and Z-type luminous sources in their hard states. Our results are a little different from the Z-type sources. Here we provide the first evidence that a similar anti-correlated feature can also be found in atoll-type source, and it is not corresponding to the lowest accretion rate.
\end{abstract}

Keywords. X-rays binary, accretion rate, cross-correlation

\section{Introduction and Data Reduction}

Most of neutron star low mass X-ray binaries can be divided into two classes viz. Z-type and atoll-type, based on their color-color diagram(CCD) or hardness-intensity diagram (HID)(Hasinger \& van der Klis 1989). For each type of source, spectral/timing states are identified which are thought to arise from different inner flow configurations (e.g., Liu et al. 2007; di Salvo et al. 2009). For atoll sources, the main three states are the extreme island state (EIS), the island state (IS), and the banana branch, the latter subdivided into lower left banana (LLB), lower banana (LB), and upper banana (UB) states. $4 \mathrm{U} 1608-52$ is a transient atoll-type source that shows outbursts with a recurrence time varying between 80 days and several years.

Anti-correlation of the soft and hard X-rays are often seen in the hard state of some BHXBs (e.g., Choudhury et al. 2005) and the horizontal branch (HB) and upper normal branch (NB) of two Z sources (Lei et al. 2008; Sriram et al. 2012). Detection of anticorrelation can help to know the radiative and geometrical structure of the accretion disk. The purpose of this work is to investigate whether the anti-correlation between the soft and hard X-rays exists as well in the atoll source of $4 \mathrm{U} 1608-52$, and its evolution.

The observations of $4 \mathrm{U}$ 1608-52 analyzed in this paper are from the Proportional Counter Array (PCA) on board the RXTE satellite during the outburst of 1998 February 3 to September 29. In this work, only PCU2 data are adopted due to the longest observational duration. The XRONOS tool "crosscor" is used for estimating the crosscorrelation between the soft X-rays $(2-3.5 \mathrm{keV})$ and the hard X-rays $(12-30 \mathrm{keV})$ for observations that containing segments longer than $2000 \mathrm{~s}$. The data of X-ray burst are excluded. For CCDs analysis, the soft and the hard colors are defined as the countrate ratio $3.5-6.0 \mathrm{keV} / 2.0-3.5 \mathrm{keV}$ and $9.7-16 \mathrm{keV} / 6.0-9.7 \mathrm{keV}$. To obtain HID, we also calculate the intensity, the count rate in the energy band $2.0-16.0 \mathrm{keV}$.

\section{Results and Discussion}

We have studied the cross-correlation of 4U 1608-52 during 1998 outburst and find that it is evolutional along the different branches, and the anti-correlation of the soft and hard X-rays is detected (Fig. 1). ObsIDs 30062-01-01-03 and 30062-01-02-05 show the obvious anti-correlations between the soft and hard X-rays. Fig. 2 shows the light 

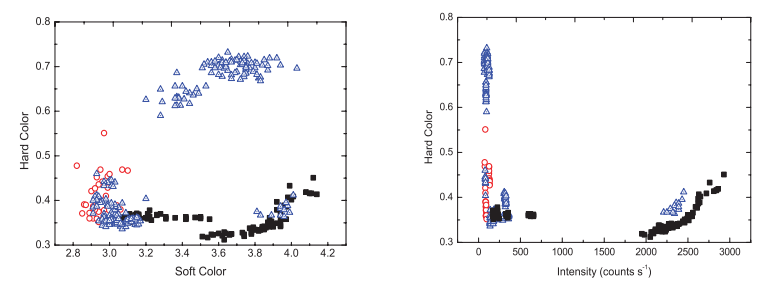

Figure 1. CCD and HID of $4 \mathrm{U}$ 1608-52 during the 1998 outburst. The square stands for the positive-correlation, the triangle corresponds to the ambiguous and the circle indicates the anti-correlation. Each point is on average of $512 \mathrm{~s}$.
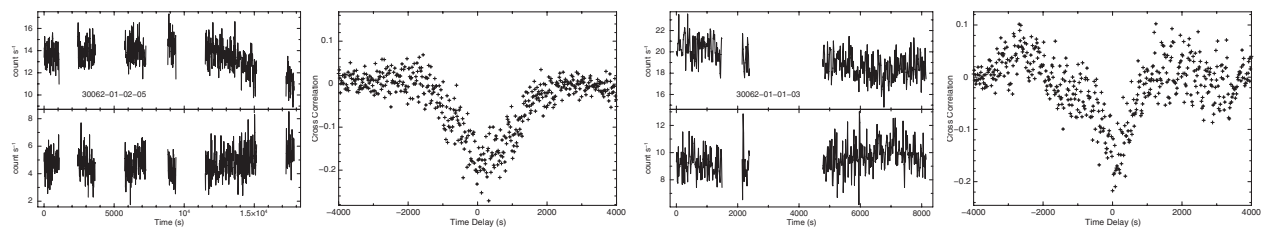

Figure 2. light curves with bin size of $16 \mathrm{~s}$ and cross-correlations of the observations with anti-correlation.

curves and cross-correlations between the soft(2.0-3.5 keV) and hard(12-30 keV) X-rays of the observations with anti-correlation detected, and no obvious time lag is detected in the two ObsIDs. In Fig. 1, we can see that, most of the observations with ambiguouscorrelation are on the IS and LLB, the others are on the UB, the observations with positive-correlation are on the LB and UB, and the observations with anti-correlation are on LLB. On the IS, there are not obvious positive or anti-correlation detected.

It has been suggested that the accretion rate is connected with the position on the CCD and HID, Our results are a little different from the Z-type sources which the anticorrelations are detected at all three branches, but mostly on the vertical HB, HB and upper NB corresponding to the lower accretion rate. For the $4 \mathrm{U} 1608-52$, there is not anti-correlated observation found in the IS corresponding to the lowest accretion rate. In the higher accretion rate(LLB), positive-correlation is dominative. In the UB which corresponds to the highest accretion rate, both the ambiguous-correlation and positivecorrelation are detected. In the low hard state of BHXBs and Z-type source, the detected anti-correlation of the soft and hard X-rays suggests the existence of a truncated accretion disk. Such truncated accretion disk could be exist in atoll-type source, however the cause of its produce is not clear in detail. The more work is needed in the future for explaining the phenomenon and physical origin by studying the relationship of the timing and spectral properties with more data.

Supported by NSFC 10903005, 11173034, 11173024 and 2009CB824800, 2012CB821800.

\section{References}

Choudhury, M., Rao, A. R., Dasgupta, S. et al. 2005, ApJ, 631, 1072

di Salvo, T., D'Aí, A., Iaria, R., et al. 2009, MNRAS, 398, 2022

Hasinger, G. \& van der Klis, M. 1989, $A \& A, 225,79$

Lei, Y. J., Qu, J. L., Song, L. M. et al. 2008, ApJ, 677, 461

Liu, Q. Z., van Paradijs, J., \& van den Heuvel, E. P. J. 2007, A\&A, 469, 807

Sriram, K., Choi, C. S., \& Rao, A. R. 2012, ApJS, 200, 16 